\title{
ANÁLISE DAS DEMONSTRAÇÕES DE RESULTADO ABRANGENTE APÓS A IMPLEMENTAÇÃO DO IFRS NO BRASIL
}

\author{
STATEMENT OF COMPREHENSIVE INCOME ANALYSIS AFTER \\ THE IMPLEMENTATION OF IFRS IN BRAZIL
}

\section{ANÁLISIS DE ESTADOS DE RESULTADO COMPLETO DESPUÉS DE LA IMPLEMENTACIÓN DE LAS IFRS EN BRASIL}

\begin{abstract}
Adolfo Henrique Coutinho e Silva
Doutor em Contabilidade e Controladoria pela Universidade de São Paulo; Professor Adjunto da Universidade Federal do Rio de Janeiro adolfocoutinho@uol.com.br
\end{abstract}

\section{Érica Gomes Rocha da Silva}

Bacharel em Ciências Contábeis pela Universidade do Estado do Rio de Janeiro ericarocha2704@yahoo.com.br

\begin{abstract}
Contextus
ISSNe 2178-9258

Organização: Comitê Científico Interinstitucional Editor Científico: Marcelle Colares Oliveira Avaliação : Double Blind Review pelo SEER/OJS Revisão: Gramatical, normativa e de formatação

Recebido em 11/04/2014

Aceito em 02/12/2014

$2^{\mathrm{a}}$ versão aceita em 18/12/2014

$3^{\text {a }}$ versão aceita em 12/01/2015

$4^{a}$ versão aceita em 30/01/2015
\end{abstract}

\section{RESUMO}

O presente estudo analisou o conteúdo informacional da Demonstração de Resultado Abrangente (DRA) publicada por 62 empresas brasileiras que compõem o Novo Mercado da BM\&FBovespa. Os resultados indicaram que: (a) aproximadamente 50\% das empresas apresentaram ganhos e perdas no resultado abrangente; (b) os efeitos líquidos dos ganhos e perdas foram positivos nos anos de 2012 e 2011 e negativos no ano de 2010; (c) os CPC 2 (Efeitos das mudanças nas taxas de câmbio e conversão de demonstrações contábeis) e CPC 38 (Instrumentos financeiros) foram os mais frequentes; e d) existem diferenças entre as métricas Resultado do Período e Resultado Abrangente para as empresas analisadas nos anos de 2011 e 2010. Os resultados observados indicam que os itens que compõem a DRA têm o potencial de gerar volatilidade no resultado do exercício caso fossem reconhecidos na Demonstração de Resultado do Exercício.

Palavras-chaves: Demonstração de resultado abrangente. Normas internacionais de Contabilidade. Relevância da informação contábil. Outros resultados abrangentes. Novo mercado. 


\begin{abstract}
This study examined the information content of the Comprehensive Income Statement published by 62 companies of "New Market" of BM \& FBovespa. The statistical analysis consisted of the application of the mean difference paired test (T test and Wilcoxon test). In addition, a qualitative analysis was performed. The results indicated that: (a) approximately $50 \%$ of companies reported gains and losses in Comprehensive Income Statement; (b) the effects of gains and losses were positive in 2012 and 2011 and negative in 2010; (c) the CPC 2 (Effects of changes in exchange rates and translation of financial statements) and CPC 38 (Financial Instruments) were the most frequent; and d) there are differences between metrics Net Income and Comprehensive Income for the companies analyzed in years 2011 and 2010. The results indicate that the items of the DRA have the potential to generate volatility in the income statement if they were recognized in the Income Statement.
\end{abstract}

Keywords: Comprehensive income statement. International Accounting standards. Relevance of accounting information. Other comprehensive income. New market.

\title{
RESUMEN
}

Este estudio examinó el contenido de la información de la Cuenta de Resultados Integral publicado por 62 empresas en el Novo Mercado de BM \& F Bovespa. El análisis estadístico consistió en la aplicación de la prueba pareada diferencia de medias (Prueba $\mathrm{T}$ y Prueba Wilcoxon). Además, se realizó un análisis cualitativo. Los resultados indicaron que (a) aproximadamente $50 \%$ de las empresas reportó ganancias y pérdidas en otros resultados integrales; (B) los efectos netos de las ganancias y pérdidas fueron positivos en los años 2012 y 2011 y negativo en 2010; (C) los CPC 2 (Efectos de las variaciones en los tipos de cambio y conversión de estados financieros) y CPC 38 (Instrumentos financieros) fueron las más frecuentes y d) existen diferencias entre las métricas Ingresos Netos y Resultado Integral para las empresas analizadas en años 2011 y 2010. Los resultados indican que los elementos de la DRA tienen el potencial de generar volatilidad en la cuenta de resultados si se les reconocen en el Estado de Resultados.

Palabras clave: Estado de resultados integral. Normas internacionales de Contabilidad. Relevancia de la información contable. Otros resultados integrales. Nuevo mercado.

\section{INTRODUÇÃO}

O Reino Unido foi o primeiro país

a exigir a divulgação do resultado abrangente com a emissão do UK Financial Reporting Standard 3(UK FRS 3) em 1992 (CAHAN et al., 2000, p. 1278). Em 1994, a Nova Zelândia passou a exigir a divulgação do resultado abrangente como parte da Demonstração das Mutações do Patrimônio Líquido (DMPL). Os EUA passaram a exigir a apresentação dos Resultados Abrangentes em 1997, quando foi emitido o SFAS 130

-Comprehensive Income.

No Brasil, a Demonstração de Resultado Abrangente deve ser elaborada de acordo com o Pronunciamento Técnico CPC 26 (Apresentação das Demonstrações Contábeis), emitido em 17/09/2009, e divulgada em conjunto com as demais demonstrações contábeis a partir do exercício de 2010. A harmonização das normas brasileiras de contabilidade com as Normas Internacionais de Contabilidade, 
emitidas pelo International Accounting Standards Board (IASB), ocorreu a partir da promulgação da Lei n. 11.638/07. O referido pronunciamento reflete basicamente o mesmo conteúdo da norma internacional, emitida pelo IASB, sobre os critérios de elaboração e divulgação da Demonstração de Resultado Abrangente.

Antes do CPC 26, não havia no Brasil normatização que tratasse do resultado abrangente e seus componentes. O CPC 26 (item 81) passou a exigir que as empresas apresentem o resultado abrangente em uma Demonstração de Resultado Abrangente separada da Demonstração de Resultado, ao passo que as normas internacionais permitem a opção pela divulgação dos resultados abrangentes junto com o resultado do período. Adicionalmente, o CPC 26 (no item 106) determinou que as entidades apresentassem a Demonstração das Mutações do Patrimônio Líquido (DMPL), informando o resultado abrangente do período.

O Pronunciamento Técnico CPC 26 estabeleceu ainda que: (a) seja divulgada, para cada componente do patrimônio líquido, a conciliação do saldo no início e no final do período, demonstrando-se separadamente as mutações decorrentes de cada item dos outros resultados abrangentes; e (b) seja apresentado, na DMPL ou nas notas explicativas, uma análise dos outros resultados abrangentes por item. Destacase que as normas contábeis emitidas pelo $\mathrm{CPC}$ as quais regulam os componentes que são registrados como outros resultados abrangentes são: CPC 02 - Efeitos de Mudanças nas Taxas de Câmbio; CPC 04 Ativo Intangível; CPC 18 Investimento em Coligada; CPC 27 - Ativo Imobilizado; CPC 32 - Tributos sobre o Lucro; CPC 33 - Benefícios a empregados; e CPC 38 Instrumentos Financeiros.

Segundo Cahan et al. (2000, p. 1273), a preocupação por parte dos órgãos reguladores com a exclusão arbitrária de alguns componentes (ganhos e perdas) da demonstração do resultado gerou a necessidade de divulgação do resultado abrangente que inclui itens que são registrados diretamente no patrimônio líquido. Notadamente, após a implementação da divulgação da Demonstração do Resultado Abrangente (DRA), os pesquisadores passaram a investigar o conteúdo informacional de tal demonstração em seu respectivo país. Em geral, a literatura internacional apresenta uma ampla discussão em relação à utilidade do Resultado do Período (Lucro Líquido do Exercício) e do Resultado Abrangente, no entanto os resultados apurados ainda são inconclusivos.

O presente estudo tem como objetivo geral analisar o conteúdo informacional da DRA publicada pelas 
empresas brasileiras de capital aberto que compõem o segmento Novo Mercado da Bolsa de Valores, Mercadorias e Futuros (BM\&FBovespa), nos anos de 2010, 2011 e 2012, após a adoção das Normas Internacionais de Contabilidade no Brasil. Mais especificamente, foi analisado: (a) se o resultado abrangente apresentou diferenças estatisticamente significativas em relação ao lucro líquido divulgado na Demonstração do Resultado do Exercício (DRE); (b) se os ajustes decorrentes dos itens que compõem o resultado abrangente foram positivos ou negativos; e (c) quais pronunciamentos contábeis mais ocasionaram efeitos no resultado abrangente divulgado pelas empresas analisadas.

Considerando a recente exigência de divulgação da DRA no Brasil, a realização do presente estudo se justifica em função da: (1) ausência de estudos realizados no Brasil que analisassem os efeitos de ganhos e perdas que passaram a ser diretamente registrados no patrimônio líquido após a adoção das normas internacionais; e da (2) escassez de estudos nacionais que tratem da relevância dos ajustes apresentados na Demonstração de Resultado Abrangente para os usuários da informação contábil.

É importante ressaltar que os resultados apurados no presente estudo apresentam aspectos peculiares da recente adoção das práticas contábeis internacionais no contexto brasileiro, sendo relevante tanto para os reguladores nacionais quanto para os reguladores internacionais, face às diferenças observadas em relação aos estudos desenvolvidos em outros países.

O restante do estudo está organizado da seguinte forma: a segunda parte apresenta a revisão de literatura, na qual são discutidos os principais estudos que trataram do tema; em seguida, são apresentados os procedimentos metodológicos e os critérios adotados para a seleção da amostra; na quarta parte, são apresentados os resultados observados na pesquisa; e, por fim, na quinta parte do estudo, é apresentada a conclusão.

\section{REVISÃO DE LITERATURA}

A literatura internacional apresenta uma grande variedade de estudos que analisaram o conteúdo informacional da DRA. Em geral, tais estudos analisaram as seguintes questões: (a) a relevância das informações de resultado abrangente e seus componentes para os usuários da informação contábil (KANAGARETNAM; MATHIEU; SHEHATA, 2009; JONES; SMITH, 2011; CAHAN et al., 2000; PRONOBIS; ZÜLCH, 2010); (b) a forma e localização das informações de resultado abrangente divulgadas pelas empresas nos países onde 
as normas contábeis sobre resultado abrangente mudaram ou foram implementadas pela primeira vez (RAHMAN; HMADAN, 2011; CHAMBERS et al., 2007; FERRARO, 2011); (c) qual componente mais impactou a DRA (RAHMAN; HMADAN, 2011); e (d) os determinantes da forma de divulgação do resultado abrangente (e seus componentes) nas demonstrações financeiras (LEE; PETRONI; SHEN, 2004; BAMBER et al., 2010).

Em relação aos estudos que examinaram a relevância do resultado abrangente (e de seus componentes), verificaram-se resultados bastante díspares como, por exemplo, os estudos de Cahan et al. (2000), Dehning e Ratliff (2004) e Kanagaretnam, Mathieu e Shehata (2009).

Cahan et al. (2000) analisaram se os componentes do resultado abrangente são mais relevantes do que o resultado do período divulgado nas demonstrações contábeis de empresas norte-americanas, bem como se a divulgação dos itens de resultado abrangente na DMPL gera mudança na relevância dos componentes de resultado abrangente em relação ao resultado do período. Os resultados do estudo não encontraram evidência de que os itens de outros resultados abrangentes fornecem informações que sejam mais relevantes que o resultado abrangente total, também não encontraram evidência de que os outros resultados abrangentes têm relevância superior ao lucro líquido após o período quando divulgado na DMPL.

Do mesmo modo, Dehning e Ratliff (2004) também concluíram que a divulgação da DRA, nos Estados Unidos (após exigência do SFAS 130 Apresentação dos Resultados Abrangentes), não aumentou a utilidade das demonstrações contábeis publicadas como um todo. Para os autores, esse resultado é consistente com a hipótese dos mercados eficientes, em que as informações disponíveis antes da exigência de divulgação da Demonstração de Resultado Abrangente não são valorizadas de forma diferente simplesmente porque elas estão sendo divulgadas de outra forma.

Por outro lado, Kanagaretnam, Mathieu e Shehata (2009), que investigaram se a exigência de empresas canadenses ( no Canadá, a obrigatoriedade de divulgação do resultado abrangente ocorreu em 2005, por exigência do The Canadian Accounting Standards Board AcSB, conforme KANAGARETNAM; MATHIEU; SHEHATA, 2009, p. 349) apresentarem a Demonstração de Resultados Abrangentes fornece ao mercado informações relevantes. Os autores concluíram que os componentes do resultado abrangente são relevantes, mas não têm tanto poder para predizer a 
rentabilidade futura, devido à sua natureza transitória.

Nos Estados Unidos, alguns pesquisadores (CHOI; ZANG, 2006; CHOI; DAS; ZANG, 2007), analisando dados com período de amostragem até 2003, argumentam que o lucro líquido prediz melhor os resultados, resultados abrangentes, e fluxos de caixa futuros do que o resultado abrangente, ao passo que Dhaliwal, Subramanyan e Trezevant (1999) afirmam que, para os anos de 1994 e 1995, o lucro líquido tem melhor poder de previsão dos fluxos operacionais de caixa e resultados futuros do que os resultados abrangentes. Biddle e Choi (2006), analisando dados de 1994 a 1998, argumentam que não há definição de qual tipo de resultados (Lucro Líquido/Resultado Abrangente) tem maior utilidade. Em relação aos estudos realizados com dados de países europeus, Wang (2006) e Goncharov e Hodgson (2008) afirmam que o lucro líquido sempre supera o resultado abrangente na previsão de desempenho (ou fluxos de caixa) futuro da empresa.

Como já destacado por Ferraro e Veltri (2012, p. 588), os estudos anteriores não têm apresentado resultados consistentes, isto é, os estudos em geral não têm encontrado suporte consistente para a relevância da DRA (formada por informações do resultado do período e informações sobre resultados abrangentes).

De acordo com Chambers et al. (2007, p. 558), uma possível razão para a falta de resultados consistentes em pesquisas anteriores é que os estudos usaram dados de períodos anteriores à data efetiva da divulgação obrigatória da DRA. Por exemplo, Dhaliwal et al. (1999) utilizaram informações sobre o resultado abrangente a partir de números dos balanços para o período em que a divulgação do resultado abrangente não era obrigatória nos EUA.

Segundo Chambers et al. (2007, p. 561), os opositores da divulgação da DRA argumentaram que o resultado abrangente possui itens que são movidos por fatores do mercado sobre os quais os gestores não têm nenhum controle e, portanto, não é uma boa base para avaliar o desempenho gerencial.

Conforme Kanagaretnam, Mathieu e Shehata (2009, p. 352), há uma visão de que o resultado do período deve medir todas as mudanças, no valor econômico de uma empresa, resultantes de todas as atividades e circunstâncias, exceto as decorrentes de investimentos $\mathrm{e}$ distribuições de dividendos aos proprietários. Esse conceito de resultado abrangente requer que as alterações de valores em todos os ativos e passivos de uma empresa fluam para a demonstração 
de resultados e sejam mensuradas a valor de mercado.

Adicionalmente, os opositores também alegam que as alterações no valor da empresa são de natureza transitória e decorrentes de atividades que não são recorrentes, tendo assim o poder de predição limitado (KANAGARETNAM; MATHIEU; SHEHATA, 2009, p. 352). Naturalmente, os argumentos sobre a irrelevância dos ganhos e perdas apresentados na DRA para os investidores e credores são utilizados como justificativa para que esses ganhos e perdas não sejam registrados na DRE, mas em uma conta do patrimônio líquido.

Por outro lado, os defensores da divulgação da DRA argumentaram que ela é a única medida que capta todas as fontes de criação de valor e que, de forma adequada, distingue criação e distribuição de valor. Ainda segundo Chambers et al. (2007, p. 561), os defensores argumentaram que a informação de resultado abrangente é uma medida que impõe disciplina sobre os gestores e analistas e que sistemas de remuneração baseados no resultado abrangente exigiriam que os gestores considerassem todos os fatores que afetam o valor da empresa, tornando, assim, menos atraente o gerenciamento de resultados.

Rahman e Hmadan (2011, p. 16) também argumentam favoravelmente à necessidade de divulgação de resultado abrangente.

O lucro de uma empresa para um determinado exercício é melhor representado pelo aumento da sua riqueza. No entanto, um aumento na riqueza da companhia pode ter ocorrido por diversas razões, algumas delas não relacionadas com as operações diárias. Por exemplo, a riqueza pode mudar por causa de flutuações nas taxas de câmbio de moedas estrangeiras, fazendo com que o valor da moeda de subsidiárias aumente ou diminua. Além disso, a riqueza pode aumentar ou diminuir devido a mudanças nas condições econômicas que alteram os valores de certos ativos e passivos. Por exemplo, as mudanças no mercado de ações podem resultar em ganhos ou perdas não realizados de retenção para investimentos detidos pela empresa. Essas mudanças na riqueza devem ser capturadas pelo resultado abrangente. (Tradução nossa)

Ao analisar a literatura sobre resultados abrangentes, é possível concluir que o tema ainda divide opiniões. De um modo geral, Pronobis e Zülch (2010, p. 7) ressaltaram que o exame do poder preditivo precisa de mais esclarecimentos. Os autores também destacaram a necessidade de realização de pesquisas sobre o poder de previsão de resultados abrangentes após a adoção do IFRS e a necessidade de realização de estudos 
dentro de cada país, devido ao fato de poder existir resultados tendenciosos considerando as particularidades de cada país.

Ao examinar a literatura sobre resultados abrangentes, foi possível verificar que também há uma grande discussão em relação à forma de divulgação do resultado abrangente e seus componentes nas demonstrações financeiras. De acordo com a literatura, existem as seguintes formas de apresentação do resultado abrangente e seus componentes: a) na Demonstração de Resultado do Período; b) na Demonstração de Resultado Abrangente; c) na Demonstração das Mutações no Patrimônio Líquido (DMPL); d) em notas explicativas; e/ou e) em uma única Demonstração de Resultado que inclua componentes do resultado do período e componentes do resultado abrangente.

Bamber et al. (2010) mencionaram que os investidores racionais processam totalmente a informação, independentemente de sua localização, de modo que não importa onde as empresas relatam o resultado abrangente. $\mathrm{Na}$ mesma linha, Dehning e Ratliff (2004, p. 229) argumentam que a simples reorganização das informações nas demonstrações financeiras não traz impacto na forma dos usuários interpretarem a informação contábil. No entanto, estudos experimentais sugerem que uma melhora na clareza da informação pode afetar a interpretação dos usuários das informações contábeis.

Segundo Tarca et al. (2008, p. 557), pesquisas anteriores concluíram que há fortes evidências de que a forma de apresentação do resultado abrangente afeta a tomada de decisão do usuário da informação contábil. Adicionalmente, os autores alegaram que a disponibilidade de informações pode não ser suficiente para garantir a tomada de decisão de forma eficaz. Os autores ainda ressaltaram que a informação deve ser colocada onde possa ser processada de forma mais eficiente por seus usuários. Assim como Tarca et al. (2008), Ferraro (2011, p. 1323) também concluiu que o formato escolhido, para divulgar o resultado e o papel atribuído aos resultados abrangentes, pode influenciar consideravelmente a avaliação dos indicadores.

$\mathrm{Na}$ mesma linha, Rahman e Hmadan (2011, p. 13) afirmam:

[...] se o valor do resultado abrangente é simplesmente enterrado na miríade de informações apresentadas nas demonstrações financeiras, sem fazer um esforço especial para fornecer a ele algum destaque, os leitores tendem a perder a importância desse número. (Tradução nossa) 
Segundo Bamber et al. (2010), o órgão regulador norte-americano, Financial Accounting Standards Board (FASB), prefere que as empresas relatem o resultado abrangente na Demonstração de Resultados, pois considera que o resultado abrangente fica mais transparente do que quando relatado na Demonstração do Patrimônio Líquido (DMPL). Dessa maneira, segue a preferência dos formuladores de normas contábeis e ajuda os gestores a ganhar uma reputação de transparência.

\section{O International Accounting} Standard Board (IASB) publicou a versão revisada do IAS 1 (Apresentação das Demonstrações Financeiras) em setembro de 2007. De acordo com Ferraro (2011, p. 1315), a referida revisão teve como objetivo também exigir a apresentação da Demonstração do Resultado Abrangente em função da influência da SFAS 130 emitida pelo FASB. Em maio de 2010, o IASB proibiu a escolha entre as duas formas de divulgação, agora as informações de resultado abrangente devem ser divulgadas em uma única demonstração dividida em itens da demonstração de resultado tradicional e itens de outros resultados abrangentes. Para o IASB, as duas alternativas reduzem a comparabilidade das demonstrações financeiras e podem criar confusão para os usuários sobre qual medida de resultado considerar (FERRARO, 2011, p. 1317).

Chambers et al.

(2007) investigaram se a forma de evidenciação das informações de outros resultados abrangentes nos EUA afeta o valor das informações do resultado abrangente no período após a emissão da SFAS 130. Os resultados do estudo sugerem que os investidores prestam mais atenção quando outros resultados abrangentes são apresentados na DMPL do que quando são apresentados na Demonstração de Resultado. Os autores argumentaram que isso pode ser atribuído ao fato de os investidores estarem mais familiarizados com o período após a implementação do SFAS 130 do que com os outros resultados abrangentes apresentados na DMPL.

No que diz respeito aos determinantes para a escolha da forma de divulgação das informações de resultado abrangente, os resultados do estudo de Bamber et al. (2010, p. 23) sugerem que as empresas com maiores ganhos e perdas não realizados sobre "ativos disponíveis para venda" são menos propensas a usar a demonstração de resultado. Além disso, Bamber et al. (2010) concluíram que empresas cujos gestores têm pagamentos baseados em ações são mais propensas a evitar a divulgação na Demonstração de Resultado. Os resultados também mostraram que os gestores com maior 
estabilidade de emprego são menos propensos a evitar a divulgação na Demonstração de Resultados.

Na Itália, Ferraro (2011) analisou como o resultado abrangente e seus componentes são divulgados nas demonstrações financeiras. Os resultados indicaram que $86 \%$ da amostra optaram por apresentar o resultado abrangente $\mathrm{e}$ seus componentes em uma Demonstração de Resultado Abrangente separada. O restante da amostra escolheu apresentar as informações em uma única demonstração de resultado abrangente. $\mathrm{O}$ autor também concluiu que as empresas as quais registraram valores negativos para o lucro líquido, mas valores positivos para outros resultados abrangentes, optaram por adotar a Demonstração de Resultado Abrangente única, a fim de dar papel intermediário para o resultado do período negativo.

Os resultados do estudo de Rahman e Hmadan (2011) indicaram que a maioria das empresas optou por apresentar a demonstração de resultado abrangente total. A conta de ajustes de conversão de moeda (Equivalente ao CPC 02 - Efeitos das Mudanças nas Taxas de Câmbio e Conversão de Demonstrações Contábeis) foi a mais apresentada pelas empresas analisadas. No que se refere aos efeitos dos itens nas contas de resultado abrangente em relação ao resultado do período, $27 \%$ das empresas foram afetadas positivamente, ao passo que $33 \%$ das empresas foram afetadas negativamente, e $39 \%$ das empresas não apresentaram componentes de outros resultados abrangentes e, portanto, não foram afetadas pelo IFRS 101.

No Brasil, destaca-se o estudo realizado por Pinheiro, Macedo e Vilamaior (2012), que analisaram a volatilidade que os Outros Resultados Abrangentes causariam no lucro líquido das empresas, caso fossem registrados no resultado do exercício. A análise compreendeu 21 empresas brasileiras que divulgaram informações contábeis na NYSE (bolsa de valores norte-americana), relativas aos exercícios de 2003 a 2007. Em resumo, os autores concluíram que não há diferença estatisticamente significativa entre as variáveis: volatilidade do Resultado Líquido e volatilidade do Resultado Abrangente, bem como verificaram que a conversão das demonstrações contábeis de investimentos no exterior é o item que mais colabora para a volatilidade identificada. Observa-se que o estudo de Pinheiro, Macedo e Vilamaior (2012) foi realizado com dados anteriores ao período da implementação das normas contábeis internacionais no Brasil.

Em resumo, os estudos relacionados com a divulgação da Demonstração do Resultado Abrangente analisam pelo menos uma das seguintes 
questões: (a) a fundamentação teórica para que os reguladores permitam que determinados ganhos e perdas transitem diretamente pelo patrimônio líquido e não sejam apresentados na Demonstração de Resultados; (b) o conteúdo e a forma de divulgação da Demonstração do Resultado Abrangente; e (c) a relevância das informações apresentadas na Demonstração do Resultado Abrangente para os usuários, especialmente investidores e credores.

Notadamente, os estudos anteriores que analisaram a relevância das informações apresentadas na Demonstração do Resultado Abrangente são inconclusivos, variando de país para país e de período analisado. Baseado em tais evidências, o presente estudo tem como objetivo analisar o conteúdo informacional da Demonstração do
Resultado Abrangente publicada pelas empresas brasileiras de capital aberto, com o intuito de avaliar se o lucro líquido e o lucro abrangente apresentam diferenças estatisticamente significativas que possam justificar a realização de estudos sobre o poder preditivo ("value relevance") dos resultados abrangentes no Brasil.

\section{METODOLOGIA}

O presente estudo consiste em uma investigação empírica quantitativa e qualitativa sobre o conteúdo e a forma de divulgação da Demonstração do Resultado Abrangente.

\subsection{Seleção da Amostra}

A Tabela 1 apresenta o grupo de empresas selecionado para o estudo, bem como demonstra a representatividade da amostra analisada.

Tabela 1 - Composição da amostra de empresas analisadas

\begin{tabular}{|c|c|c|}
\hline & $\underline{\mathrm{N}}$ & $\underline{\%}$ \\
\hline Quantidade de empresas do segmento Novo Mercado da BM\&FBovespa ${ }^{(1)}$ & 126 & $100,0 \%$ \\
\hline ( - ) Empresas financeiras, seguradoras e corretoras, e equivalentes & $\underline{(6)}$ & $\underline{4,8 \%}$ \\
\hline ( =) Empresas pesquisadas (população) & 120 & $95,2 \%$ \\
\hline Quantidade de empresas na amostra & 62 & $51,7 \%$ \\
\hline
\end{tabular}

Nota: (1) Dados coletados no site da BM\&FBovespa, em setembro de 2012. (2) As empresas de capital aberto podem pertencer a segmentos de listagem com regras rígidas e diferenciadas de governança corporativa, tais como: a) Novo Mercado; b) Nível 2; c) Nível 1; e d) Bovespa Mais. Fonte: elaborada pelos autores.

A amostra deste estudo é formada por empresas que compõem o segmento Novo Mercado da Bolsa de Valores,
Mercadorias e Futuros (BM\&FBovespa), que é composta por 126 empresas. A escolha do referido segmento foi realizada 
pela expectativa de a qualidade das informações divulgadas no Relatório Anual dessas empresas apresentarem, em geral, uma qualidade superior, possibilitando uma adequada coleta de dados para realização do estudo. Destacase que o segmento de Novo Mercado da BM\&FBovespa é composto por empresas que atendem aos padrões mais elevados de governança corporativa dentre as empresas de capital aberto com registro na Comissão de Valores Mobiliários.

Segundo Silva, Silva e Laurencel (2014), algumas empresas ingressam em segmentos diferenciados de governança corporativa com o propósito de aumentar sua credibilidade no mercado mobiliário. Assim, na medida do aumento das exigências por mais informações financeiras, essas empresas estariam dispostas a aumentar seus níveis de transparência. Analisando o nível de transparência referente ao Ativo Imobilizado (CPC 28), para o ano de
2012, Silva, Silva e Laurencel (2014) argumentam que o segmento diferenciado de mercado (Bovespa Mais, Níveis 1 e 2 de Governança Corporativa e Novo Mercado) apresenta um índice de não divulgação menor que o segmento tradicional de mercado.

Depois de selecionado o segmento de empresas a serem estudadas, foram excluídas as instituições financeiras, seguradoras e corretoras, instituições de intermediação para operações do mercado de capitais e empresas de intermediação financeira que apresentam características significativamente diferentes. Após as exclusões, a população de empresas objeto do estudo ficou restrita a 120 empresas.

Por fim, foi realizada a seleção de uma amostra probabilística (aleatória) de 62 empresas, que apresentam características de todas as empresas que compõem o segmento Novo Mercado. A tabela abaixo apresenta a composição da amostra por setor de atividade. 
Tabela 2 - Composição da amostra por setor de atividade

$\begin{array}{llll} & \text { Setor de atividade } & \text { Quant. de empresas } & \text { \% } \\ 1 & \text { Agro e Pesca } & 3 & 5 \% \\ 2 & \text { Alimentos e Bebidas } & 4 & 6 \% \\ 3 & \text { Comércio } & 5 & 8 \% \\ 4 & \text { Construção } & 6 & 10 \% \\ 5 & \text { Eletroeletrônicos } & 1 & 2 \% \\ 6 & \text { Energia Elétrica } & 3 & 5 \% \\ 7 & \text { Máquinas Indústrias } & 1 & 2 \% \\ 8 & \text { Minerais Não Metálicos } & - & 0 \% \\ 9 & \text { Mineração } & 2 & 3 \% \\ 10 & \text { Papel e Celulose } & 1 & 2 \% \\ 11 & \text { Petróleo e Gás } & 3 & 5 \% \\ 12 & \text { Siderurgia \& Metalurgia } & 2 & 3 \% \\ 13 & \text { Software e Dados } & 1 & 2 \% \\ 14 & \text { Telecomunicações } & - & 0 \% \\ 15 & \text { Transporte Serviços } & 5 & 8 \% \\ 16 & \text { Têxtil } & 3 & 5 \% \\ 17 & \text { Veículos e peças } & 2 & 3 \% \\ 18 & \text { Outros } & \underline{20} & \underline{32 \%} \\ \text { Total } & & 62 & 100 \%\end{array}$

Nota: (a) Foi utilizada a classificação setorial da Economática (C).

Fonte: elaborada pelos autores.

\subsection{Coleta e Análise de Dados}

A coleta das informações foi realizada manualmente, em fevereiro de 2014, através de pesquisa dos dados disponíveis nas Demonstrações Financeiras Padronizadas (DFPs) dos anos de 2012, 2011 e 2010 arquivadas no site da Comissão de Valores Mobiliários (CVM). A análise se restringiu aos anos de 2010, 2011 e 2012, pois o estudo buscou analisar as mudanças nos resultados abrangentes após o período de adoção das Normas Internacionais de Contabilidade no Brasil.

A coleta dos dados (e a respectiva análise do conteúdo) compreendeu a pesquisa das seguintes partes integrantes das Demonstrações Financeiras Padronizadas (DFPs): (a) Demonstração de Resultados Abrangentes; (b) Demonstração das Mutações do Patrimônio Líquido; (c) Demonstração de Resultado do Exercício; e (d) Notas Explicativas.

A análise qualitativa ocorreu por meio da análise das notas explicativas das 
demonstrações contábeis das empresas analisadas para: (a) identificar as empresas que apresentaram informações de resultado abrangente; e (b) identificar como essas informações foram apresentadas, bem como os componentes do resultado abrangente que impactaram as empresas.

Com a finalidade de analisar se o resultado abrangente foi estatisticamente diferente do resultado do período, foi realizado o teste de diferença de médias (paramétrico ou não paramétrico) para análise de amostras pareadas, isto é, os testes foram realizados utilizando-se $\mathrm{o}$ Lucro Líquido (LL), divulgado na Demonstração do Resultado do Exercício, e o Resultado Abrangente (LLA), que inclui os ajustes divulgados na Demonstração de Resultado Abrangente.

Para análise da adequação do teste estatístico paramétrico (teste t) ou não paramétrico (teste Wilcoxon), foi realizado o teste estatístico Kolmogorov-Smirnov para análise da normalidade dos dados. A Tabela 3 apresenta as estatísticas descritivas das variáveis estudadas.

Tabela 3 - Estatísticas Descritivas das Variáveis LL e LLA (Valores em Reais Mil)

Painel 1 - Empresas impactadas (com ajustes na DRA)

\begin{tabular}{|c|c|c|c|c|c|c|}
\hline & \multicolumn{2}{|l|}{2012} & \multicolumn{2}{|l|}{2011} & \multicolumn{2}{|l|}{2010} \\
\hline & $\underline{\mathrm{LL}}$ & $\underline{\mathrm{LLA}}$ & $\underline{\mathrm{LL}}$ & $\underline{\mathrm{LLA}}$ & $\underline{\mathrm{LL}}$ & $\underline{\mathrm{LLA}}$ \\
\hline Média & 148.387 & 164.859 & 91.268 & 123.833 & 201.873 & 186.568 \\
\hline DP & 565.266 & 580.905 & 349.201 & 358.183 & 274.064 & 263.541 \\
\hline Máximo & 2.320 .618 & 2.325 .597 & 830.901 & 840.809 & 744.050 & 739.577 \\
\hline Mínimo & -1.172 .774 & -1.162 .893 & -746.683 & -605.532 & -155.005 & -155.783 \\
\hline $\mathrm{N}$ & 31 & & 27 & & 26 & \\
\hline
\end{tabular}

Painel 2 - Todas as empresas da amostra

\begin{tabular}{|c|c|c|c|c|c|c|}
\hline & \multicolumn{2}{|l|}{2012} & \multicolumn{2}{|l|}{2011} & \multicolumn{2}{|l|}{2010} \\
\hline & $\underline{\mathrm{LL}}$ & $\underline{\text { LLA }}$ & $\underline{\mathrm{LL}}$ & $\underline{\text { LLA }}$ & $\underline{\mathrm{LL}}$ & $\underline{\text { LLA }}$ \\
\hline Média & 154.043 & 162.279 & 151.393 & 165.807 & 250.667 & 244.144 \\
\hline DP & 622.747 & 629.765 & 460.357 & 461.721 & 406.197 & 404.159 \\
\hline Máximo & 2.320 .618 & 2.325 .597 & 1.816 .915 & 1.816 .915 & 1.830 .914 & 1.830 .914 \\
\hline Mínimo & -2.172 .855 & -2.172 .855 & -905.189 & -905.189 & -155.005 & -155.783 \\
\hline $\mathrm{N}$ & 62 & & 61 & & 61 & \\
\hline
\end{tabular}

Nota: (a) Definição das siglas: (1) LL - Lucro Líquido do período; (2) LLA - Lucro Abrangente; (3) DP Desvio Padrão. (b) Uma empresa não apresentou as demonstrações financeiras nos anos de 2011 e 2010. Fonte: elaborada pelos autores. 
Buscando verificar se o resultado abrangente foi estatisticamente diferente do resultado do período, foram formuladas as seguintes hipóteses estatísticas:

$$
\mathbf{H}_{\mathbf{0}} \text { Não há diferença }
$$
estatisticamente significativa entre $\mathrm{o}$ Resultado Abrangente (LLA) e o Resultado do Período (LL) das empresas que compõem o segmento do Novo Mercado;

$\mathbf{H}_{1}$ : Há diferença estatisticamente significativa entre o Resultado Abrangente (LLA) e o Resultado do Período (LL) das empresas que compõem o segmento do Novo Mercado.

A análise dos impactos do Resultado Abrangente (e seus componentes) sobre o resultado do período (lucro líquido) também foi realizada por meio da análise da magnitude dos efeitos (em termos monetários e percentuais) dos ajustes dos componentes que compõem o resultado abrangente. Assim como realizado no estudo de Rahman e Hmadan (2011), foi examinado se as variações do resultado do período após os itens que formam $\mathrm{o}$ resultado abrangente foram positivas ou negativas (ou seja, se resultado abrangente é maior ou menor que o resultado do período). Por fim, foi analisado qual pronunciamento contábil mais afetou o resultado abrangente das empresas analisadas.

\section{RESULTADOS}

\subsection{Evidenciação das informações do resultado abrangente pelas empresas analisadas}

As informações gerais sobre a forma de apresentação da Demonstração do Resultado Abrangente são apresentadas, a seguir, na Tabela 4 .

O Painel 1 da Tabela 4 demonstra que quase a totalidade das empresas analisadas divulgou a Demonstração de Resultado Abrangente, sendo que, em 2010 (primeiro ano de divulgação), quatro empresas não apresentaram a DRA. Em 2011 e 2012, apenas uma empresa estava na mesma situação. Considerando que não foi observado se havia ressalva nos pareceres de auditoria externa, é possível depreender que as empresas não foram impactadas por ajustes de ganhos e perdas no resultado abrangente no período.

Conforme análise do Painel 2 da Tabela 4, percebe-se que, no geral, a maior parte das empresas analisadas apresentaram os Outros Resultados Abrangentes (ORA) tanto na Demonstração de Resultados Abrangentes (DRA) quanto na Demonstração das Mutações do Patrimônio Líquido (DMPL), obedecendo assim o exigido no CPC 26. Em relação às empresas que não apresentaram a Demonstração de Resultado Abrangente, no período 
analisado, apenas uma empresa alegou não apresentar saldos nas contas que compõem a Demonstração de Resultado Abrangente. No que se refere às Notas Explicativas, nos dois primeiros anos de elaboração obrigatória da DRA, poucas empresas apresentaram informações adicionais sobre o resultado abrangente, sendo que apenas 5 empresas em 2011 e 6 empresas em 2010 apresentaram quadros com informações sobre itens do resultado abrangente. Entre essas empresas, a maior parte delas explicou a forma de reconhecimento e mensuração, enquanto as demais não apresentaram informações. Em 2012, esse número aumentou para 10 empresas.

Tabela 4 - Apresentação das informações de Resultado Abrangente

\begin{tabular}{|c|c|c|c|c|c|c|}
\hline \multicolumn{7}{|c|}{ Painel 1 - Apresentação da Demonstração de Resultado Abrangente (DRA) } \\
\hline & $\underline{2012}$ & & $\underline{2011}^{\mathrm{a}}$ & & $\underline{2010}^{\mathrm{a}}$ & \\
\hline & Quant. & $\underline{\%}$ & Quant. & $\underline{\%}$ & Quant. & $\underline{\%}$ \\
\hline Empresas que apresentaram a DRA & 61 & $98,4 \%$ & 60 & $98,4 \%$ & 57 & $93,4 \%$ \\
\hline Empresas que não apresentaram DRA & $\underline{1}$ & $\underline{1,6 \%}$ & $\underline{1}$ & $\underline{1,6 \%}$ & $\underline{4}$ & $\underline{6,6}$ \\
\hline Total & 62 & $100,0 \%$ & 61 & $100,0 \%$ & 61 & $100,0 \%$ \\
\hline
\end{tabular}

Painel 2 - Forma de divulgação dos Resultados Abrangentes nas DFPs ${ }^{\mathrm{b}}$

$\begin{array}{llllllll} & \underline{2012} & & \underline{2011} & & \underline{2010} & \\ & \underline{\text { Quant. }} & \underline{\%} & \underline{\text { Quant. }} & \underline{\%} & \underline{\text { Quant. }} & \underline{\%} \\ \text { Divulgação somente na DRA } & 1 & 1,6 \% & 2 & 3,3 \% & 1 & 1,8 \% \\ \text { Divulgação na DRA e na DMPL }^{\mathrm{d}} & \underline{60} & \underline{98,4 \%} & \underline{58} & \underline{96,7 \%} & \underline{56} & \underline{98,2 \%} \\ \text { Total }^{\text {Tol }} & 61 & & 100,0 \% & 60 & 100,0 \% & 57 & 100,0 \%\end{array}$

Painel 3 - Empresas com ganhos e perdas registrados em Resultado Abrangente no período ${ }^{\text {b }}$

$\begin{array}{llllllll} & \underline{2012} & & \underline{2011} & & \underline{2010} & \\ & \underline{\text { Quant. }} & \underline{\%} & \underline{\text { Quant. }} & \underline{\%} & \underline{\text { Quant. }} & \underline{\%} \\ \text { Apresentaram DRA c/ajustes } & 31 & 50,8 \% & 27 & 45,0 \% & 26 & 45,6 \% \\ \text { Apresentaram DRA s/ajustes } & \underline{30} & \underline{49,2 \%} & \underline{33} & \underline{55,0 \%} & \underline{31} & \underline{54,4 \%} \\ \text { Total } & 61 & 100,0 \% & 60 & 100,0 \% & 57 & 100,0 \%\end{array}$

Nota: (a) Uma das empresas selecionadas não arquivou suas demonstrações contábeis no site da CVM nos anos de 2011 e 2010, razão pela qual a amostra ficou menor. (b) Inclui apenas as empresas que apresentaram a Demonstração de Resultados Abrangentes. (c) Demonstrações Financeiras Padronizadas (DFP). (d) Demonstração das Mutações do Patrimônio Líquido $\quad$ (DMPL). Fonte: elaborada pelos autores.

Em relação aos impactos dos contábeis publicadas, observa-se, no Painel resultados abrangentes nas demonstrações 3 (da Tabela 4), que aproximadamente 
metade das empresas não apresentou movimentação na DRA $(45,6 \%$ em 2010 , 45,0\% em 2011 e 50,8\% em 2012). Tal constatação é importante na medida em que demonstra que um significativo número de empresas não estava sujeito aos tratamentos contábeis que geram o reconhecimento de ganhos e perdas diretamente no patrimônio líquido.

A Tabela 5 apresenta os itens com saldo nas Demonstrações de Resultado Abrangente das empresas analisadas.

Tabela 5 - Quantidade de ajustes por empresa no resultado abrangente das empresas analisadas

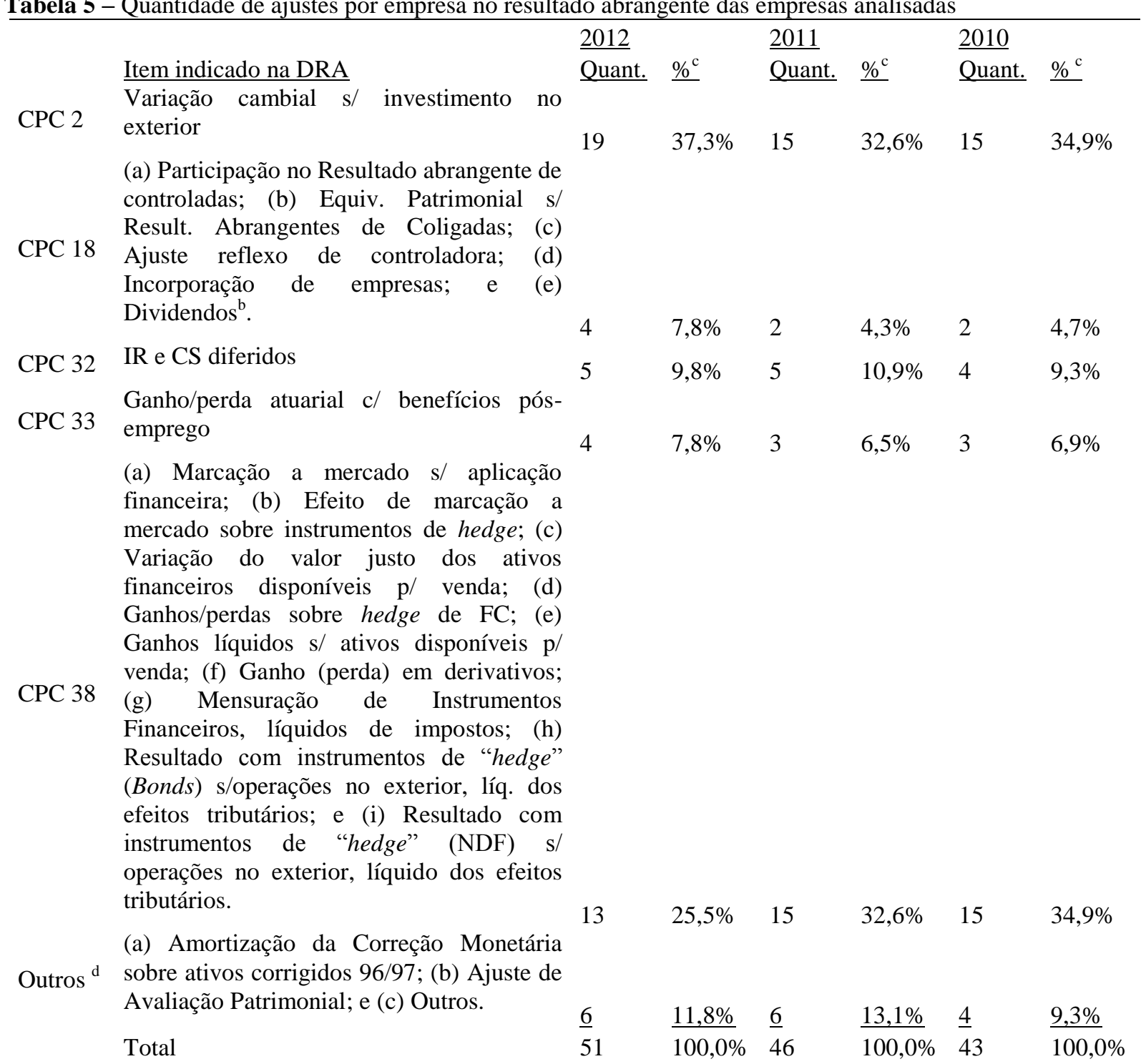

Nota: (a) Quant.: corresponde ao número de empresas que apresentaram saldos decorrentes dos respectivos CPCs nas contas de resultado abrangente. (b) O efeito da conta dividendos demonstrado na DRA não impacta a conta Ajuste de Avaliação Patrimonial, mas sim o Patrimônio Líquido. É importante destacar que o estudo utilizou informações consolidadas, por isso aparece a conta Dividendos. (c) quantidade de ajustes sobre o total de ajustes identificado. (d) Empresas que não forneceram informações que permitissem identificar claramente qual pronunciamento técnico estava relacionado ao item identificado.

Fonte: elaborada pelos autores. 
A partir da análise dos dados apresentados na Tabela 5, verifica-se que o Pronunciamento Técnico CPC 2 foi o item que mais frequentemente impactou o resultado abrangente das empresas no período analisado. Observa-se que o item Variação Cambial sobre Investimento no Exterior (CPC 02) impactou 19 empresas $(37,3 \%)$ em 2012, 15 empresas $(32,6 \%)$ em 2011 e 15 empresas $(34,9 \%)$ em 2010. Esse resultado é similar aos resultados encontrados nos estudos de Rahman e Hmadan (2011) e Bamber et al. (2010), nos quais o item variação cambial foi $o$ componente mais apresentado pelas empresas analisadas.

O segundo pronunciamento técnico mais recorrente foi o CPC 38, que trata do reconhecimento e mensuração de Instrumentos Financeiros. Destaca-se que esse item apresentou uma grande variedade de termos utilizados. Notadamente, a grande variedade de nomenclaturas utilizadas dificulta a compreensão, comparação e análise das Demonstrações do Resultado Abrangente, tanto por parte do pesquisador quanto pelo usuário da informação.

\subsection{Efeitos das informações do resultado abrangente no resultado das empresas analisadas}

A Tabela 6 apresenta os resultados do teste de diferença (pareada) de médias do Lucro Líquido e do Lucro Líquido Abrangente, para as empresas da amostra que apresentaram ajustes nas contas que compõem a Demonstração de Resultado Abrangente e para a amostra total, de modo a verificar se existem diferenças estatisticamente significativas entre as duas métricas contábeis, nos anos de 2010, 2011 e 2012 .

Tabela 6 - Resultados dos Testes Estatísticos de Diferença de Médias entre o Lucro Líquido e o Lucro Abrangente do Período

Painel 1 - Apenas empresas com ajustes na DRA

\begin{tabular}{|c|c|c|c|c|c|c|c|c|c|c|}
\hline & & & & Variação & $\underline{\mathrm{KS}^{\mathrm{c}}}$ & & $\underline{\text { Teste } \mathrm{T}^{\mathrm{d}}}$ & & $\underline{\text { Wilcox }}$ & \\
\hline Ano & $\underline{\mathrm{N}^{\mathrm{o}}}$ & $\underline{\mathrm{LL}^{\mathrm{a}}}$ & $\underline{L L A}^{a}$ & $\%^{\mathrm{b}}$ & $\underline{Z}$ & $\underline{\text { Sig. }}$ & $\underline{\mathrm{t}}$ & $\underline{\text { Sig. }}$ & $\underline{Z}$ & Sig. \\
\hline 2012 & 31 & 148.387 & 164.859 & $+11,1 \%$ & $\begin{array}{l}1,155 \\
1,171\end{array}$ & $\begin{array}{l}0,139 \\
0,129\end{array}$ & $-0,992$ & 0,329 & $-1,117$ & 0,264 \\
\hline 2011 & 27 & 91.268 & 123.833 & $+35,7 \%$ & $\begin{array}{l}0,640 \\
0,579\end{array}$ & $\begin{array}{l}0,808 \\
0,891\end{array}$ & $-1,270$ & 0,215 & $-2,114$ & $0,034 * *$ \\
\hline 2010 & 26 & 201.873 & 186.568 & $-7,6 \%$ & $\begin{array}{l}1,260 \\
1,040\end{array}$ & $\begin{array}{l}0,084 * * * \\
0,230\end{array}$ & 1,264 & 0,218 & $-2,781$ & $0,005^{*}$ \\
\hline
\end{tabular}


Tabela 6 - Resultados dos Testes Estatísticos de Diferença de Médias entre o Lucro Líquido e o Lucro Abrangente do Período - continuação

Painel 2 - Amostra total

\begin{tabular}{|c|c|c|c|c|c|c|c|c|c|c|}
\hline & & & & Variação & $\underline{\mathrm{KS}^{\mathrm{c}}}$ & & Teste $\mathrm{T}^{\mathrm{d}}$ & & Wilcox & \\
\hline$\underline{\text { Ano }}$ & $\underline{\mathrm{N}^{\mathrm{o}}}$ & $\underline{\mathrm{LL}^{\mathrm{a}}}$ & $\underline{L L A}^{a}$ & $\%^{\mathrm{b}}$ & $\underline{Z}$ & $\underline{\text { Sig. }}$ & $\underline{\mathrm{t}}$ & $\underline{\text { Sig. }}$ & $\underline{Z}$ & Sig. \\
\hline 2012 & 62 & 154.043 & 162.279 & $+5,3 \%$ & $\begin{array}{l}1,702 \\
1,749\end{array}$ & $\begin{array}{l}0,006 * \\
0,004 *\end{array}$ & $-0,992$ & 0,325 & $-1,117$ & 0,264 \\
\hline $2011^{\mathrm{d}}$ & 61 & 151.393 & 165.807 & $+9,5 \%$ & $\begin{array}{l}1,594 \\
1,566\end{array}$ & $\begin{array}{l}0,012 * * \\
0,015 * *\end{array}$ & $-1,262$ & 0,212 & $-2,114$ & $0,034 * *$ \\
\hline $2010^{d}$ & 61 & 250.667 & 244.144 & $-2,6 \%$ & $\begin{array}{l}2,003 \\
1,894\end{array}$ & $\begin{array}{l}0,001 * \\
0,002 *\end{array}$ & 1,255 & 0,214 & $-2,781$ & $0,005^{*}$ \\
\hline
\end{tabular}

Notas: (a) Valores em R\$ Mil, sendo os valores médios do total dos saldos de lucro líquido (LL) e dos saldos de resultado abrangente (LLA - Lucro Líquido Abrangente) de todas as empresas com ajustes na DRA, no caso do Painel 1, e de toda a amostra, exceto a empresa que não divulgou a demonstração financeira, no caso do Painel 2; (b) Diferença entre o LLA e o LL dividido pelo LL; (c) Teste Kolmogorov-Smirnov (KS) para análise da normalidade das variáveis. A primeira linha refere-se ao LL, enquanto a segunda linha refere-se ao LLA; (d) Teste paramétrico ( $t$ test) de diferença de médias pareadas (paired samples test); (e) Teste não paramétrico (wilcoxon test) de diferenças de médias pareadas; (f) As significâncias dos testes estatísticos apresentadas correspondem à probabilidade nas duas caudas (2-tailed); e (g)* 1\% de nível de significância, ** 5\% de nível de significância, *** $10 \%$ de nível de significância.

Fonte: elaborada pelos autores.

Com a finalidade de verificar a existência de diferença significativa entre o Resultado do Período (LL) e o Resultado Abrangente (LLA), foram realizados testes estatísticos de diferenças de médias (paramétrico - $T$ test e não paramétrico Wilcoxon test), conforme o resultado da normalidade apurado para as distribuições analisadas (conforme indicado pelo teste Kolmogorov Smirnov).

Os resultados apurados na análise estatística das empresas que apresentaram ajustes na Demonstração do Resultado Abrangente (vide Painel 1 da Tabela 6) demonstram que apenas no ano de 2010 o Resultado do Período (LL) e o Resultado
Abrangente (LLA) são estatisticamente diferentes $(\mathrm{z}=-2,781, \mathrm{p}$-value $<1 \%)$.

Analisando as diferenças entre o Resultado do Período (LL) e o Resultado Abrangente (LLA) para toda a amostra (painel 2 da Tabela 6), observa-se que existem diferenças estatisticamente significativas nas referidas variáveis para os anos de 2011 e 2010 ( $\mathrm{z}=-2,114$, pvalue $<5 \%$ e $\mathrm{z}=-2,781, \mathrm{p}$-value $<1 \%$, respectivamente).

De fato, pode-se constatar que os resultados estatísticos não paramétricos, para a amostra completa e para as empresas que apresentaram ajustes na DRA, foram exatamente iguais, isto é, o resultado não é afetado pela inclusão das empresas que não apresentaram ajustes. 
Observa-se também que, no teste paramétrico, a inclusão das empresas sem ajustes na DRA afeta a análise da normalidade das distribuições, especialmente no ano de 2011.

Em resumo, os resultados apurados permitem concluir que existem diferenças entre as métricas Resultado do Período (LL) e Resultado Abrangente (LLA) para as empresas que compõem o Novo Mercado nos anos de 2011 e 2010. As diferenças médias apuradas foram de $+9,5 \%$ e $-2,6 \%$ em 2011 e 2010, respectivamente, quando consideradas todas as empresas da amostra; e de $+35,7 \%$ e $-7,6 \%$ em 2011 e 2010 , respectivamente, quando consideradas apenas as empresas que apresentaram ajustes na DRA. Os resultados encontrados indicam que os efeitos são relevantes quando considerado apenas o grupo de empresas com ajustes na
Demonstração do Resultado Abrangente do período.

O exercício de 2012 apresentou uma variação nas métricas de $+11,1 \%$ e $+5,3 \%$, para a amostra parcial e completa, respectivamente, mas ambas as diferenças não são estatisticamente significativas (tanto no teste paramétrico quanto no não paramétrico).

Assim, pode-se afirmar também que os efeitos dos ganhos e perdas reconhecidos diretamente no patrimônio líquido (e indicados na DRA) variam significativamente de ano para ano, tanto em termos percentuais quanto na direção do efeito.

A Tabela 7 demonstra a natureza dos ajustes das contas do Resultado Abrangente no resultado do período das empresas analisadas no período analisado.

Tabela 7 - Natureza dos ajustes do Resultado Abrangente ${ }^{\text {a }}$

\begin{tabular}{|c|c|c|c|c|c|c|}
\hline \multirow[b]{2}{*}{$\underline{\text { Natureza dos ajustes }}^{\mathrm{b}}$} & \multicolumn{2}{|l|}{2012} & \multicolumn{2}{|l|}{2011} & \multicolumn{2}{|l|}{2010} \\
\hline & Quant. & $\underline{\%}$ & Quant. & $\underline{\%}$ & Quant. & $\underline{\%}$ \\
\hline Positivo & 17 & $54,8 \%$ & 20 & $74,1 \%$ & 5 & $19,2 \%$ \\
\hline Negativo & $\underline{14}$ & $45,2 \%$ & $\underline{7}$ & $25,9 \%$ & $\underline{21}$ & $\underline{80,8 \%}$ \\
\hline Subtotal & 31 & $100,0 \%$ & 27 & $100,0 \%$ & 26 & $100,0 \%$ \\
\hline Sem ajuste $^{c}$ & $\underline{31}$ & & $\underline{34}$ & & $\underline{35}$ & \\
\hline Total & 62 & & 61 & & 61 & \\
\hline
\end{tabular}

\footnotetext{
Notas: (a) Inclui apenas as empresas que arquivaram as demonstrações contábeis na Comissão de Valores Mobiliários, razão pela qual o número de empresas é menor em 2011 e 2010, conforme indicado na Tabela 3; (b) As empresas foram segregadas em dois grupos, sendo o primeiro daquelas que apuraram um ajuste total positivo, isto é, os efeitos aumentaram o lucro líquido do período da empresa analisada. O segundo grupo é formado pelas demais empresas; e (c) Inclui as empresas que não divulgaram a DRA, conforme indicado no painel 1 da Tabela 3. Consideram-se essas empresas como sem ajuste em função de apresentarem o parecer dos auditores independentes sem ressalva ou ênfase.
}

Fonte: elaborada pelos autores. 
Como pode ser observada na Tabela 7, a distribuição entre empresas com ajustes positivos e negativos variou significativamente de ano para ano. Em 2010, a maior parte das empresas $(80,8 \%)$ teve ajuste negativo, enquanto, no ano de 2011, a maior parte das empresas $(74,1 \%)$ teve ajuste positivo. Em 2012, observa-se um equilíbrio entre os dois grupos. Tal resultado talvez possa decorrer dos efeitos cíclicos dos efeitos contábeis, quando as variações cambiais ou variações de preços de mercado de ativos financeiros, por exemplo, apresentam crescimento e redução ao longo do tempo.

A maior quantidade de impactos negativos evidenciados em 2010 pelas empresas analisadas coincide com os resultados encontrados por Ferraro (2011) no estudo das empresas italianas listadas na bolsa de valores no ano de 2009. Uma diferença relevante do presente estudo em relação ao estudo realizado por Ferraro (2011) refere-se ao fato de que nenhuma empresa da amostra analisada pela referida autora alegou não ter sido impactada (ou não apresentou informações) em relação ao resultado abrangente.

A Tabela 8 permite visualizar como cada um dos pronunciamentos técnicos emitidos pelo CPC acarretou ajustes na Demonstração de Resultado Abrangente no período analisado.

Tabela 8 - Efeito dos ajustes no resultado abrangente do período (31 empresas em 2012; 27 empresas em 2011; e 26 empresas em 2010)

Painel 1 - Magnitude dos Ajustes (\%) por CPC no Resultado Abrangente

\begin{tabular}{|c|c|c|c|c|c|c|c|}
\hline$\underline{\text { Ano }}$ & $\underline{\text { Efeito }}$ & $\underline{\mathrm{CPC}} 2$ & $\underline{\mathrm{CPC} 18}$ & $\underline{\mathrm{CPC}} 32$ & $\underline{\mathrm{CPC}} 33$ & $\underline{\mathrm{CPC}} 38$ & $\underline{\text { Outros }}$ \\
\hline \multirow[t]{2}{*}{2012} & $\%$ do ajuste ${ }^{a}$ & $160,4 \%$ & $-0,3 \%$ & $16,9 \%$ & $-71,3 \%$ & $-14,5 \%$ & $8,7 \%$ \\
\hline & Média $\%$ dos ajustes & $8,4 \%$ & $-0,1 \%$ & $3,4 \%$ & $-17,8 \%$ & $-1,1 \%$ & $1,5 \%$ \\
\hline \multirow[t]{2}{*}{2011} & $\%$ do ajuste & $105,7 \%$ & $1,1 \%$ & $5,9 \%$ & $-8,7 \%$ & $-7,0 \%$ & $2,9 \%$ \\
\hline & Média $\%$ dos ajustes & $7,0 \%$ & $0,6 \%$ & $1,2 \%$ & $-2,9 \%$ & $-0,5 \%$ & $0,5 \%$ \\
\hline \multirow[t]{2}{*}{2010} & $\%$ do ajuste & $-109,5 \%$ & $-3,5 \%$ & $-14,7 \%$ & $-23,9 \%$ & $51,6 \%$ & $0,00 \%$ \\
\hline & Média \% dos ajustes & $-7,3 \%$ & $-1,7 \%$ & $-3,7 \%$ & $-8,0 \%$ & $3,4 \%$ & $0,00 \%$ \\
\hline
\end{tabular}


Tabela 8 - Efeito dos ajustes no resultado abrangente do período (31 empresas em 2012; 27 empresas em 2011; e 26 empresas em 2010) - continuação

Painel 2 - Natureza do Ajuste (em quantidade) por CPC no Resultado Abrangente

\begin{tabular}{|c|c|c|c|c|c|c|c|}
\hline$\underline{\text { Ano }}$ & Natureza do Ajuste & CPC 2 & $\underline{\mathrm{CPC}} 18$ & CPC 32 & CPC 33 & CPC 38 & $\underline{\text { Outros }}$ \\
\hline \multirow{3}{*}{2012} & Positivo & 13 & 2 & 4 & 0 & 3 & 4 \\
\hline & Negativo & $\underline{6}$ & $\underline{2}$ & $\underline{1}$ & $\underline{4}$ & $\underline{10}$ & $\underline{2}$ \\
\hline & Total & 19 & 4 & 5 & 4 & 13 & 6 \\
\hline \multirow{3}{*}{2011} & Positivo & 12 & 2 & 3 & 1 & 10 & 4 \\
\hline & Negativo & $\underline{3}$ & $\underline{0}$ & $\underline{2}$ & $\underline{2}$ & $\underline{5}$ & $\underline{2}$ \\
\hline & Total & 15 & 2 & 5 & 3 & 15 & 6 \\
\hline \multirow{3}{*}{2010} & Positivo & 2 & 0 & 1 & 0 & 7 & 2 \\
\hline & Negativo & $\underline{13}$ & $\underline{2}$ & $\underline{3}$ & $\underline{3}$ & $\underline{8}$ & $\underline{2}$ \\
\hline & Total & 15 & 2 & 4 & 3 & 15 & 4 \\
\hline
\end{tabular}

Notas: (a) O percentual do ajuste foi calculado a partir da divisão do valor total resultante de um CPC pelo total de ajustes; (b) A média do percentual dos ajustes corresponde à divisão do percentual dos efeitos pela quantidade de ajustes de cada CPC; e (c) A quantidade do Painel 2 corresponde à contagem simples das ocorrências dos ajustes na Demonstração de Resultado Abrangente.

Fonte: elaborada pelos autores.

No que se refere à magnitude dos ajustes, o Pronunciamento Técnico CPC 02 (Efeitos das Mudanças nas Taxas de Câmbio e Conversão de Demonstrações Contábeis) apresentou o maior efeito, confirmando os resultados apresentados no referencial teórico. O percentual do ajuste foi de 160,4\% em 2012, 105,7\% em 2011 e $-109,5 \%$ em 2010.

Destaca-se também o efeito significativo do Pronunciamento Técnico CPC 33 (Benefícios a Empregados) em
2012, com efeito negativo de $71,3 \%$ e do Pronunciamento Técnico CPC 38 em 2010, com efeito positivo de 51,6\%. Notadamente, a cada ano, observa-se uma variação significativa na contribuição de cada pronunciamento do $\mathrm{CPC}$ na composição do Lucro Abrangente.

A Tabela 9 apresenta os efeitos no Lucro Abrangente (apenas das empresas com ajustes na DRA) em termos monetários para cada um dos anos analisados. 
Tabela 9 - Efeitos dos ajustes do resultado abrangente por CPC (em termos monetários)

\begin{tabular}{|c|c|c|c|c|c|c|c|c|c|}
\hline \multirow[b]{2}{*}{$\underline{\mathrm{CPC}}$} & \multicolumn{3}{|c|}{2012} & \multicolumn{3}{|c|}{2011} & \multicolumn{3}{|c|}{$\underline{2010}$} \\
\hline & $\underline{\mathrm{R} \$}$ & Quant. & Média & $\underline{\mathrm{R} \$}$ & Quant. & Média & $\underline{\mathrm{R} \$}$ & Quant. & Média \\
\hline CPC 2 & 819.042 & 19 & 43.107 & 929.064 & 15 & 61.938 & -435.793 & 15 & -29.053 \\
\hline CPC 18 & -1.323 & 4 & -331 & 9.998 & 2 & 4.999 & -13.826 & 2 & -6.913 \\
\hline CPC 32 & 86.421 & 5 & 17.284 & 52.252 & 5 & 10.450 & -58.575 & 4 & -14.644 \\
\hline СРC 33 & -363.988 & 4 & -90.997 & -76.131 & 3 & -25.377 & -95.019 & 3 & -31.673 \\
\hline СРC 38 & -74.094 & 13 & -5.700 & -61.778 & 15 & -4.119 & 205.303 & 15 & 13.687 \\
\hline Outros & $\underline{44.568}$ & $\underline{6}$ & $\underline{7.428}$ & 25.845 & $\underline{6}$ & $\underline{4.308}$ & $\underline{-17}$ & $\underline{4}$ & $\underline{-4.25}$ \\
\hline Total & 510.626 & 51 & -29.209 & 879.250 & 46 & 52.199 & -397.927 & 43 & -68.600 \\
\hline
\end{tabular}

Notas: (a) Valores em Reais Mil; (b) CPC 02 (R2) - Efeitos das mudanças nas taxas de câmbio e conversão de demonstrações contábeis, CPC 18 (R2) - Investimento em coligada, em controlada e em empreendimento controlado em conjunto, CPC 32 - Tributos sobre o lucro, CPC 33 (R1) - Benefícios a empregados e CPC 38 Instrumentos Financeiros: reconhecimento e mensuração; e (c) Inclui apenas empresas com ajustes na DRA. Fonte: elaborada pelos autores.

Em termos monetários, o Pronunciamento Técnico CPC 2 foi o que mais afetou a DRA das empresas nos anos analisados, sendo os efeitos de R $\$ 819.042$ mil em 2012, R\$ 929.064 mil em 2011 e negativo em R\$ 435.793 mil em 2010. Destaca-se que o Pronunciamento Técnico CPC 33 gerou ajuste negativo em todos os anos (R\$ 363.988 mil em 2012, R\$ 76.131 mil em 2011 e R\$ 95.019 mil em 2010).

A análise dos efeitos no Resultado Abrangente por setor de atividade demonstra que o CPC 02 (CPC que mais impactou as empresas analisadas) apresentou efeitos relevantes nos setores de: (a) Veículos e Peças; (b) Alimentos e Bebidas; (c) Mineração; e (d) Petróleo e Gás (somente em 2012). O Pronunciamento Técnico CPC 38 afetou mais o Resultado Abrangente das empresas que compõem o setor de Veículos e Peças, Agropecuária e Pesca e Outros.

\section{CONCLUSÃO}

Considerando a recente exigência de divulgação da Demonstração de Resultado Abrangente (DRA) no Brasil, o presente estudo teve como objetivo principal analisar o conteúdo informacional da DRA publicada pelas empresas brasileiras de capital aberto que compõem o segmento Novo Mercado da Bolsa de Valores, Mercadorias e Futuros (BM\&FBovespa) nos anos de 2010, 2011 e 2012, após a adoção das Normas Internacionais de Contabilidade no Brasil. Mais especificamente, foi analisado: (a) se o resultado abrangente apresentou diferenças estatisticamente significativas em relação ao lucro líquido divulgado na Demonstração do Resultado do Exercício 
(DRE); (b) se os itens que impactaram o resultado abrangente foram positivos ou negativos; e (c) quais pronunciamentos contábeis mais impactaram o resultado abrangente divulgado pelas empresas analisadas. Para analisar o conteúdo informacional da Demonstração de Resultado Abrangente, foram utilizados testes estatísticos de diferenças de médias para amostras pareadas (Lucro Líquido do período - LL e o Resultado Abrangente LLA), bem como uma análise qualitativa dos componentes da DRA em cada período.

Em resumo, os resultados apresentados na análise dos dados demonstraram que:

(a) Aproximadamente metade das empresas não apresentou movimentação na DRA $(45,6 \%$ em 2010, $45,0 \%$ em 2011 e 50,8\% em 2012);

(b) Poucas empresas apresentaram informações adicionais em notas explicativas sobre $\mathrm{o}$ resultado abrangente (sendo 6 empresas em 2010, 5 empresas em 2011 e 10 empresas em 2012);

(c) Os Pronunciamentos Técnicos que mais frequentemente impactaram o resultado abrangente das empresas no período analisado foram os CPC 2 - Efeitos das Mudanças nas Taxas de Câmbio e Conversão de Demonstrações Contábeis (sendo 19 empresas, 37,3\%, em
2012, 15 empresas, 32,6\%, em 2011 e 15 empresas, 34,9\%, em 2010) e o CPC 38 Reconhecimento e Mensuração de Instrumentos Financeiros;

(d) O Pronunciamento Técnico CPC 02 apresentou o maior efeito, confirmando os resultados apresentados em estudos anteriores (160,4\% em 2012, $105,7 \%$ em 2011 e $-109,5 \%$ em 2010). Destaca-se também o efeito significativo do Pronunciamento Técnico CPC 33 (Benefícios a Empregados) no ano de 2012 (efeito negativo de 71,3\%) e do Pronunciamento Técnico CPC 38 em 2010 (efeito positivo de $51,6 \%$ );

(e) A distribuição entre empresas com impactos positivos $\mathrm{e}$ negativos variou significativamente de ano para ano. Em 2010, a maior parte das empresas $(80,8 \%)$ teve ajuste negativo, enquanto, no ano de 2011, a maior parte das empresas $(74,1 \%)$ teve ajuste positivo. Em 2012, observa-se um equilíbrio entre os dois grupos; e

(f) Os resultados apurados permitem concluir que existem diferenças entre as métricas Resultado do Período (LL) e Resultado Abrangente (LLA) para as empresas que compõem o Novo Mercado nos anos de 2011 e 2010. As diferenças médias apuradas foram de $+9,5 \%$ em 2011 e $-2,6 \%$ em 2010, quando consideradas todas as empresas da amostra; e de $+35,7 \%$ em 2011 e $-7,6 \%$ em 
2010, quando consideradas apenas as empresas que apresentaram ajustes na DRA. Os resultados encontrados indicam que os efeitos são relevantes quando considerado apenas o grupo de empresas impactadas, isto é, com ajustes na Demonstração do Resultado Abrangente do período.

Os resultados sugerem que os efeitos provocados pelos novos pronunciamentos técnicos no Resultado Abrangente podem variar significativamente de empresa para empresa e de ano para ano, tanto em relação à magnitude do efeito quanto na direção do efeito, conforme já sugerido pelos estudos anteriores (RAHMAN; HMADAM, 2011). Tal resultado pode decorrer dos efeitos cíclicos dos ajustes contábeis, quando as variações cambiais ou variações de preços de mercado de ativos financeiros, por exemplo, apresentam crescimento e redução ao longo do tempo (como sugerido por KANAGARETNAM; MATHIEU; SHEDATA, 2009).

Notadamente, a grande variedade de nomenclaturas adotada para os componentes decorrentes da aplicação do CPC 38 (Instrumentos Financeiros) dificulta a compreensão, comparação e análise das Demonstrações do Resultado Abrangente, tanto por parte do pesquisador quanto pelo usuário da informação. Assim, os órgãos reguladores poderiam emitir orientações (OCPC) específicas sobre a Demonstração do Resultado Abrangente, de modo a ampliar a qualidade das informações prestadas pelas empresas, inclusive no que diz respeito à comparação entre empresas.

Os resultados apurados permitem concluir que, para as empresas de capital aberto que pertencem ao segmento de Novo Mercado da BM\&FBovespa, um significativo número de empresas não estavam sujeitas aos tratamentos contábeis que geram o reconhecimento de ganhos e perdas diretamente no patrimônio líquido (50\% em 2012, 55,7\% em 2011 e 57,3\% em 2010). Por outro lado, existem empresas que apresentaram grandes diferenças entre o Lucro Líquido e o Resultado Abrangente e, consequentemente, devem merecer atenção dos investidores (acionistas e credores) e dos profissionais que realizam as análises das demonstrações contábeis preparadas pelas empresas. Notadamente, como os efeitos decorrentes dos CPCs podem apresentar magnitudes e sinais diferentes, é razoável supor que eles possam ser relevantes (individualmente ou em conjunto) para os usuários da informação contábil quando analisados de forma segregada.

Também é importante destacar que os resultados observados indicam que os itens que compõem a Demonstração de 
Resultados Abrangentes têm o potencial de gerar volatilidade nos resultados do exercício se forem reconhecidos na Demonstração de Resultado do Exercício.

Espera-se que os resultados observados no presente estudo contribuam para a literatura nacional e internacional, na medida em que demonstram como a DRA, exigida nas normas internacionais e utilizada em diversos países, é utilizada, e evidencia o impacto dos ganhos e perdas que não transitam pela Demonstração de Resultado no contexto brasileiro.

A principal limitação observada no presente estudo deve-se ao fato de que somente foram analisadas as empresas que compõem o segmento Novo Mercado, o que não possibilita a generalização dos resultados para empresas que não pertencem a esse grupo. Ressalta-se que a escolha da análise do referido grupo de empresas deveu-se à premissa de que essas empresas apresentam uma maior qualidade das informações evidenciadas, permitindo a realização do estudo com base em dados confiáveis.

Considerando que, em alguns períodos, o Resultado Abrangente possa apresentar valores significativamente diferentes do Lucro Líquido do período, recomenda-se que estudos futuros: (1) analisem amostras pertencentes a outros segmentos da BM\&FBovespa; examinem como os componentes da demonstração abrangente afetam os indicadores econômicos e financeiros das empresas; e (3) verifiquem a relevância dessas informações para os usuários da informação contábil através de estudos quantitativos (value relevance) $\mathrm{e}$ qualitativos (questionários e entrevistas).

\section{REFERÊNCIAS}

BAMBER, Linda Smith; JIANG, John Xuefeng; PETRONI, Kathy R.; WANG, Isabel Yanyan. Comprehensive Income: who's afraid of performance reporting? Accounting Review, California, v. 85, n. 1, p. 97-126, 2010.

BM\&FBOVESPA.Disponível em: $<$ http://www.bmfbovespa.com.br>. Acesso em: 22 dez. 2012.

CAHAN, Steven F.; COURTENAY, Stephen M.; GRONEWOLLER, Paul L.; UPTON, David R. Value relevance of mandated comprehensive income disclosures. Journal of Business Finance and Accounting, v. 27, n. 9-10, p. $1273-$ 1301, nov./dez.. 2000.

CHAMBERS, Dennis; LINSMEIER, Thomas J.; SHAKESPEARE, Catherine; SOUGIANNIS, Theodore. An evaluation of SFAS n.130 comprehensive income disclosures. Review of Accounting Studies, v. 12, n. 4, p. 557-593, dez.. 2007.

COMITÊ DE PRONUNCIAMENTOS CONTÁBEIS. CPC 00 (R1)-Estrutural conceitual para elaboração e divulgação de Relatório Contábil Financeiro. Disponível em: <http://www.cpc.com.br/>. Acesso em: 15 jan.2014.

CVM- Comissão de Valores Mobiliários. Disponível em: <http://www.cvm.gov.br/>. Acesso em: 10 abr. 2013. 
DEHNING, Bruce; RATLIFF, Paulette A. Comprehensive Income: evidence on the effectiveness of FAS 130. The Journal of American Academy of Business, Cambridge, v. 4, n. 1-2, p. 228-232, mar., 2004.

DHALIWAL, D.; SUBRAMANYAM, K. R.; TREZEVANT, R. Is comprehensive income superior to net income as a measure of firm perfomance. Journal of Accounting and Economics, v. 26, p. 4367, 1999.

FERRARO, Olga. Comprehensive income in Italy: reporting preferences and its effects on performance indicators. Journal of Modern Accounting and Auditing, v. 7, n. 12, p. 1315-1328, dez.. 2011.

FERRARO, Olga; VELTRI, Stefania. A critical analysis of the empirical researches on comprehensive income value relevance. European Journal of Scientific Research, v. 76, n. 4, p. 587-594, 2012.

JONES, Denise A.; SMITH, Kimberly J. Comparing the value relevance, predictive value, and persistence of other comprehensive income and special items. The Accounting Review, v. 86, n. 6, p. 2047-2073, 2011.

KANAGARETNAM, Kiridaran; MATHIEU, Robert; SHEHATA, Mohamed. Usefulness of comprehensive income reporting in Canada. Journal of Accounting and Public Polic, v. 28, n. 4, p. 349-365, jul./ago. 2009.

LEE, Yen-Jung; PETRONI, Kathy R.; SHEN, Min. Cherry picking, financial reporting quality, and comprehensive income reporting choices: the case of property-liability insurers. . Disponível em: $<$ http://papers.ssrn.com/sol3/papers.cfm?a bstract_id=450660>. Acesso em:01 dez.2012.

PINHEIRO, L. E. T.; MACEDO, R. P.; VILAMAIOR, A. G. Lucro Líquido versus
Lucro Abrangente: Uma análise empírica da volatilidade. Revista Universo Contábil, v. 8, n. 4, p. 06-18, out./dez., 2012.

PRONOBIS, Paul; ZÜLCH, Henning. The Predictive power of comprehensive income and its individual components under IFRS. Disponível em: http://papers.ssrn.com/sol3/papers.cfm?abs tract_id=1576384. Acesso em: 01 dez.2012.

\section{COMITÊ DE PRONUNCIAMENTOS} CONTÁBEIS. CPC 26 - Apresentação das Demonstrações Contábeis. Disponível em: < http://www.cpc.org.br>. Acesso em:08 fev. 2013.

RAHMAN, Azhar Abdul; HMADAN, Mohd Diah $\mathrm{Hj}$. Comprehensive Income: reporting preferences of malaysian listed companies on the ACE Market. 2011. Disponível em: <http://wbiconpro.com/315-Azhar.pdf>. Acesso em: 01 dez.2012.

REES, Lynn; SHANE, Phil. Academic research and standard setting: the case of other comprehensive income. Accounting Horizons, v. 26, n. 4, p. 1-27.dez.., 2012.

SILVA, J. S.; SILVA, A. H. C.; LAURENCEL, L. C.. Nível de evidenciação obrigatória nas empresas de capital aberto: uma análise do CPC 27. In: Congresso ANPCONT, 8, 2014, Rio de Janeiro. Anais... Rio de Janeiro, 2011.

TARCA, A.; BROWN, P.; HANCOCK, P.; WOODLIFF, D.; BRADBURY, M.; ZIJL, T. V.. Identifying decision useful information with the matrix format income statement. Journal of International Financial Management and Accounting, v. 19, n. 2, p. 184-217, jun., 2008. 